Christovam Barcellos 1,2

\title{
Distribuição espacial da leptospirose no Rio Grande do Sul, Brasil: recuperando a ecologia dos estudos ecológicos
}

\author{
Spatial distribution of leptospirosis \\ in Rio Grande do Sul, Brazil: recovering \\ the ecology of ecological studies
}

Célia Beatriz Lammerhirt 2

Marco Antonio B. de Almeida 2

Edmilson dos Santos 2

\footnotetext{
1 Departamento de Informações em Saúde, Centro de Informação Científica e Tecnológica, Fundação Oswaldo Cruz. Av. Brasil 4365, Rio de Janeiro, $R J$ 21045-900, Brasil. xris@fiocruz.br

2 Centro de Vigilância Ambiental, Secretaria Estadual de Saúde do Rio Grande do Sul. Rua Domingos Crescêncio 132, Porto Alegre, $R S$ 90650-090, Brasil.
}

\begin{abstract}
Leptospirosis is an endemic disease in the State of Rio Grande do Sul, Brazil, and presents a broad diversity of exposure routes, reservoirs, etiological agents, and clinical features. The main objective of this work is to identify transmission areas and possible ecological components of leptospirosis transmission. This was accomplished through the aggregation of epidemiological data into spatial units that represent the State's socio-environmental diversity. The 1,274 confirmed leptospirosis cases that occurred in 2001 were georeferenced in the counties of residence. The county maps were overlaid on environmental units characterizing land use, altitude, and river basins. Incidence rates for each environmental class were calculated, along with their statistical significance, through GIS aggregation operations. The highest incidence rates were verified in coastal sedimentary areas with low altitude and predominantly agricultural land use. In these areas, most of the cases were associated with irrigated farming. The results suggest the existence of favorable ecological characteristics for leptospirosis transmission in places involving proliferation of peri-domiciliary rodents and intensive agricultural production. The article discusses the effects of data aggregation into environmental units, as well as strategies to control the endemic in the State.
\end{abstract}

Key words Leptospirosis; Ecology; Surveillance; Geographic Information System

Resumo A leptospirose apresenta no Estado do Rio Grande do Sul uma grande diversidade de situações de exposição, reservatórios, agentes etiológicos e quadros clínicos. O principal objetivo desse trabalho é identificar áreas de maior risco e possíveis componentes ecológicos da transmissão da leptospirose por meio da agregação de dados epidemiológicos em unidades espaciais que representem a diversidade sócio-ambiental do Estado. Os 1.274 casos confirmados de leptospirose ocorridos em 2001 foram georreferenciados por município de residência. Os mapas de municípios foram sobrepostos aos de caracterização de uso do solo, relevo e bacias hidrográficas. Com as operações de SIG, foram calculadas as taxas de incidência para cada categoria e sua significância estatística. As maiores taxas de incidência foram verificadas em áreas sedimentares litorâneas, de baixa altitude e uso do solo predominantemente agrícola. Nessas áreas, a maior parte dos casos está associada à lavoura irrigada. Os resultados sugerem a existência de características ecológicas favoráveis à transmissão da leptospirose em locais de proliferação de roedores sinantrópicos e de produção agrícola intensiva. São discutidos os efeitos da agregação de dados em unidades ambientais na análise de dados epidemiológicos e estratégias de controle da endemia no Estado.

Palavras-chave Leptospirose; Ecologia; Vigilância; Sistemas de Informações Geográficas 


\section{Introdução}

A leptospirose é uma doença aguda e endêmica em grande parte do mundo, transmitida por bactérias da espécie Leptospira interrogans mediante contato com solo ou água contaminados. O ciclo de transmissão da leptospirose envolve a interação entre reservatórios animais, um ambiente favorável e grupos humanos suscetíveis. Os fatores de risco associados à infecção dependem, portanto, de características da organização espacial, dos ecossistemas e das condições de vida e trabalho da população (Murhekar et al., 1998). Os diferentes sorovares circulantes produzem uma grande diversidade de situações de exposição, reservatórios e quadros clínicos. Por exemplo, os ratos (R. norvegicus e $R$. ratus) são os principais reservatórios da L. icterohemorrhagiae no meio urbano, enquanto cães e gado são reservatórios de $L$. canicola e L. hardjo, dominantes no meio rural. Um número crescente de casos tem sido observado no mundo, associados a atividades esportivas e de lazer, o que aumenta a suspeita de um ciclo silvestre da doença (Katz et al., 1991; Morgan et al., 2002). Um dos maiores desafios dos estudos da leptospirose tem sido a tipificação de ambientes e modos de transmissão, que são altamente dependentes dos sorovares.

O Estado do Rio Grande do Sul apresenta uma alta incidência de leptospirose, com cerca de 10 casos por 100 mil habitantes, superior à média do país $(3,5$ casos por 100 mil habitantes). A maior parte dos casos (86\%) corresponde a pessoas do sexo masculino e de residentes na zona rural (69\%). O local provável de infecção, indicado pela investigação epidemiológica de casos, aponta tanto o ambiente de trabalho quanto o domicílio como principais formas de contato com o agente.

A epidemiologia estuda a distribuição dos eventos relacionados com o processo saúde-doença na população. Os chamados "estudos ecológicos” representam uma estratégia da epidemiologia para a análise de dados de grupos populacionais, por intermédio da qual se compara a freqüência de doença no tempo ou no espaço. Segundo alguns autores clássicos da epidemiologia, esses estudos têm caráter apenas descritivo, sem a capacidade de testar de hipóteses (Morgenstern, 1982). O uso de grupos humanos e não o indivíduo como unidade de análise tem gerado críticas às possíveis generalizações de resultados (Susser, 1994a). Por outro lado, vários trabalhos têm apontado a utilidade e até necessidade dos estudos ecológicos, particularmente no contexto das doenças infectocontagiosas (Schwartz, 1994; Susser, 1994b). A
Ecologia é definida como o estudo da totalidade ou padrão de relações entre organismos e seu ambiente, por isso a abordagem ecológica está fortemente associada ao conceito de lugar. Os estudos ecológicos na Epidemiologia buscam, portanto, captar determinantes de saúde, que fogem ao âmbito do indivíduo, mas dizem respeito a coletividades (Susser, 1994b).

As unidades espaciais de agregação de dados epidemiológicos têm sido, por imposição dos sistemas de informação, os diversos níveis da administração pública, como o município ou o estado. Os processos tanto ambientais quanto sociais, que promovem ou restringem situações de risco à saúde, não estão limitados a estas fronteiras administrativas. Outras formas de estratificação e visualização de indicadores em mapas devem ser buscadas. Segundo Openshaw (1984), uma das formas de controlar a influência de fatores de confusão nos estudos ecológicos pode ser a escolha correta de unidades de agregação de dados.

Neste trabalho são analisados os resultados de distribuição espacial da leptospirose no Rio Grande do Sul, utilizando-se diferentes recortes espaciais que correspondem a zoneamentos ecológicos. Este estudo é viabilizado pela agregação em diversas unidades espaciais com o uso de Sistemas de Informações Geográficas (SIG).

\section{Metodologia}

Todos os casos de leptospirose foram investigados de acordo com roteiro baseado em ficha de investigação e confirmados pelo teste sorológico de macro-aglutinação ou critérios clínico-epidemiológicos (FUNASA, 2001). Os 1.274 casos confirmados e notificados ocorridos em 2001 foram georreferenciados conforme seu município de residência. Os mapas de municípios foram sobrepostos aos de caracterização de uso do solo, relevo e bacias hidrográficas. As seguintes camadas de dados foram utilizadas para a agregação de dados epidemiológicos: uso do solo (IBGE, 1986) e faixas de altitude e bacias hidrográficas (ANEEL, 2000). Esses mapas foram gerados valendo-se da classificação de imagens de satélite, complementadas por trabalhos de campo para o levantamento de características de vegetação, geomorfologia, hidrografia e pedologia, constituindo uma das primeiras aproximações para a identificação de regiões naturais do estado (Pereira, 2000). O uso de imagens de satélite e outros instrumentos de sensoriamento remoto para a vigilância de doenças tem sido feito com modelos que rela- 
cionam tipos de vegetação e clima com a presença de vetores, o que é intermediado pela presença de fontes de alimentação e abrigo (Mills \& Childs, 1998).

Por operações booleanas em ambiente SIG, foram somados os números de casos de leptospirose e da população residente dentro dos limites de cada categoria dos mapas. No caso do limite do município ser interceptado por mais de uma classificação de uso do solo, faixa de altitude ou bacia hidrográfica, considerou-se o número de casos de leptospirose e de população proporcional à área da interseção entre esses polígonos. Foram então recalculadas as taxas de incidência para cada categoria desses mapas e sua significância estatística, obtida pela comparação entre o número de casos observados e esperados. Assume-se como hipótese nula do teste que o número esperado em cada área é proporcional à população, segundo uma distribuição de Poisson (Bailey \& Gratell, 1995; Kulldorff \& Nagarwalla, 1995).

\section{O problema das unidades espaciais de agregação}

A adoção de unidades espaciais, por definição discretas e independentes, tem sido uma das estratégias mais utilizadas, e criticadas, dos estudos ecológicos (Nurminen, 1995). Nessas unidades são integrados dados tanto ambientais, quanto sociais e epidemiológicos, procurandose correlações entre eles. As unidades políticoadministrativas são, em geral, adotadas como territórios de referência para a construção desses indicadores. $\mathrm{O}$ estabelecimento de escalas de trabalho na epidemiologia, como na cartografia, é reconhecidamente artificial. O espaço geográfico é contínuo e constituído por um sistema de objetos e ações com inúmeras articulações verticais e horizontais (Santos, 1999). Neste espaço se manifestam variáveis globais de ação local e outros processos de origem local com pequena amplitude, com resultados também locais. A organização de redes no espaço permite, cada vez mais, que estes processos sejam simultâneos e abrangentes. Do ponto de vista econômico, as escalas de ação global, regional, local estão intrinsecamente ligadas e são interdependentes, constituindo uma hierarquia espacial de forças. Também para os fenômenos chamados físicos, a natureza possui escalas hierárquicas, estudadas pela ecologia da paisagem (Forman \& Godron, 1986). Desta maneira, processos geológicos globais produzem condições de solo que podem se modificar em função de fenômenos regionais de clima, em uma estrutura hierárquica cuja interação de forças forma uma paisagem local única.

A escala cartográfica é uma forma de representação da superfície da terra, enquanto a escala geográfica seria aquela do recorte do evento estudado. Na cartografia, a passagem de uma para outra escala envolve um processo de generalização, simplificação ou eliminação de alguns objetos, de modo a facilitar a visualização de informações, ao mesmo tempo em que surgem novas informações antes ignoradas. Este processo não é realizado automaticamente, mediante diminuição das distâncias representadas pelo mapa, mas, ao contrário, é realizado intelectualmente, pela seleção de informações que podem compor a nova escala de trabalho de modo coerente com os objetivos de seu estudo (Monmonier, 1996).

A necessidade de se restringir a extensão do estudo e a lógica de coleta de dados epidemiológicos impõem uma escala de trabalho, definida a partir do momento da escolha de unidades espaciais de referência. Com o aprimoramento dos sistemas de informação, particularmente dos SIG, incrementou-se a possibilidade de produzir diferentes formas de agregação de dados, construindo-se indicadores em diferentes unidades espaciais conforme o interesse de pesquisa. Um mesmo ponto (evento de saúde) pode estar contido em diferentes tipos de unidades espaciais: um bairro, uma bacia hidrográfica, um distrito sanitário, etc., definidos por polígonos nos mapas (Vine et al., 1997). Para que haja uma relação unívoca entre ponto e polígono, as unidades espaciais devem ser contíguas, configurando-se uma malha de unidades espaciais discretas. A utilização de técnicas de suavização de dados espaciais pode dissolver os limites previamente estabelecidos entre unidades espaciais (Bailey \& Gratell, 1995). A concepção de unidades espaciais como áreas homogêneas baseia-se na delimitação de um território a partir da uniformidade de certas características onde os critérios e objetivos de trabalho indicarão as variáveis a serem utilizadas para regionalização (Piquet et al., 1986).

Dentre os critérios para a escolha de unidades espaciais de referência destacam-se: a presença e qualidade do registro destas unidades nos bancos de dados, o reconhecimento da unidade espacial por parte da população, a existência de grupos populacionais organizados e de instâncias administrativas na unidade (Barcellos \& Santos, 1997). Além disso, para fins de pesquisa e diagnóstico de situação, a divisão territorial proposta deve apresentar uma máxima homogeneidade interna e heterogeneidade externa das unidades, de modo a apontar gradi- 
entes de risco. O trabalho em pequenas unidades espaciais, por um lado, traz uma maior precisão na localização de eventos, o que pode aperfeiçoar estimativas de exposição (Vine et al., 1997). Por outro lado, a agregação de dados epidemiológicos e demográficos em unidades maiores reduz um efeito de instabilidade de taxas, aumentando tanto sua base populacional (denominador) quanto a probabilidade de ocorrência de eventos (numerador). Essa agregação pode falsear informações, construindo grandes médias que apagam diferenciais internos (Carvalho \& Cruz, 1998). Estatisticamente, existe um tamanho e uma divisão ótima da área de abrangência em unidades de análise que permitam: evidenciar diferenciais entre regiões sem uniformizá-las; aumentar o poder estatístico de discernimento; e finalmente, estabilizar taxas de morbi-mortalidade, maximizando os valores do numerador e do denominador (Morris \& Munasinghe, 1993).

O efeito da agregação de dados espaciais sobre resultados estatísticos tem sido estudado há longo tempo (Gehlke \& Biehl, 1934, apud Laurent \& Thomas, 1997). Os diferentes resultados obtidos segundo os recortes espaciais foi chamado de problema da unidade de área modificável (Openshaw, 1984). A maior parte destes trabalhos mostra que as correlações aumentam à medida que se agregam os dados em unidades espaciais maiores. Essa tendência parece ser mais forte quando os dados originais estão autocorrelacionados no espaço (Bailey \& Gratell, 1995). Os indicadores epidemiológicos apresentam diferentes variações consoante a escala de análise. Segundo Cleek (1979), as maiores variabilidades de taxas sugerem a existência de dependência espacial e a atuação de fatores de risco ambientais, cabendo aos estudos ecológicos a busca de níveis de agregação de dados em que as variabilidades são maiores.

\section{A leptospirose segundo diferentes recortes ecológicos do Estado do Rio Grande do Sul}

A Figura 1 mostra as taxas de incidência de leptospirose nos municípios do Rio Grande do Sul. São verificadas altas taxas de incidência em diversas regiões do Estado, não permitindo estabelecer um padrão de distribuição espacial da endemia. A distribuição das taxas de incidência nos 467 municípios do Estado apresenta uma grande variabilidade de valores ( 0 a 401 casos por 100 mil habitantes), observando-se uma maioria de municípios (327) com nenhum caso confirmado. A média de 12,5 casos por $100 \mathrm{mil}$ habitantes e desvio-padrão de 40,9 indicam a variabilidade dos resultados e a forte assimetria da sua distribuição. Somente 17 municípios apresentaram um número de casos significativamente superior ao valor esperado, pelo teste de Poisson, conforme assinalado na Figura 1. Esses municípios estão localizados principalmente na região central e sul do Estado. Na região norte, onde predominam municípios com pequena área e população, as taxas de incidência não apresentam significância estatística.

As tabelas mostram os resultados obtidos de número de casos e taxa de incidência de leptospirose no Estado segundo as diferentes classificações das unidades ambientais. Foram também calculadas as populações residentes e área de cada unidade espacial. Com base nessas tabelas foram calculados os números de casos observados e esperados. Esses valores foram comparados e verificada sua significância estatística pelo teste de Poisson.

A Tabela 1 mostra a incidência de leptospirose segundo unidades de uso do solo. As áreas com atividades agrícolas surgem na análise com um número de casos confirmados significativamente superior ao esperado. Essas áreas se situam ao longo do litoral, da depressão central e região noroeste do Estado. Com exceção da última, essas áreas são sujeitas a inundações e a sua drenagem permitiu sua transformação em lavouras irrigadas de arroz (IBGE, 1986; Rambo, 1994). É importante salientar que a detecção de culturas agrícolas pelas imagens de satélite ou radar foi realizada por meio da identificação de amplas áreas contíguas que apresentam a mesma característica ótica. Diversas monoculturas vêm se ampliando no Estado, como a da soja, que ocupa grande parte da região noroeste, que, todavia, se diferencia das áreas de cultivo de arroz pela intensa mecanização da produção. As áreas com atividades agrícolas cobrem um total de 37,9\% do território do Estado e são responsáveis por $68,5 \%$ do total de casos de leptospirose. Nessas áreas é alta a proporção de casos relacionados ao ambiente de trabalho (42,4\%), enquanto nas demais áreas é de apenas $12,4 \%$. Também nas áreas de atividades agrícolas, há predominância do ambiente rural na transmissão da doença $(74,3 \%)$, enquanto nas demais áreas, os ambientes rurais são responsáveis por apenas $37,3 \%$ dos casos.

As áreas com vegetação preponderantemente arbustiva ou de campo (estepe e savana) apresentam baixas taxas de incidência da doença. $\mathrm{O}$ Pampa, por exemplo, é dominado por vegetação baixa, sobretudo as gramíneas, e elementos arbóreos encontrados em matas ciliares. As chuvas são mais esparsas em relação ao restante do 
Taxa de incidência de leptospirose nos municípios do Rio Grande do Sul, Brasil. Os círculos claros mostram municípios com taxa de incidência significativamente superior à média no Estado.

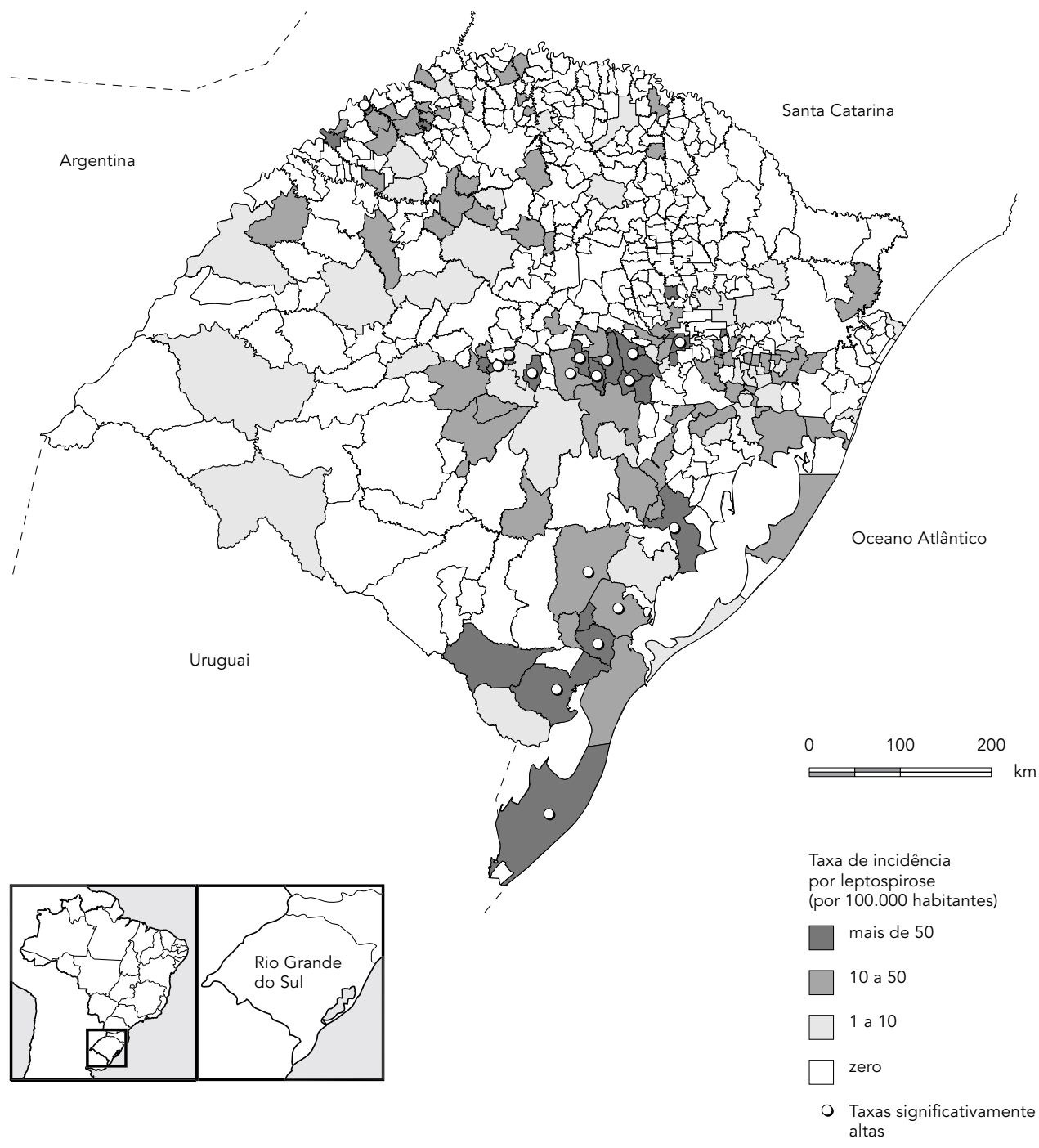

Estado. Essas áreas são caracterizadas pela criação extensiva de gado, com baixa ocupação humana no campo. Esse padrão de baixa transmissão da doença se repete nas áreas com florestas ombrófilas, remanescentes da Mata Atlântica.

A variação da incidência de leptospirose segundo faixas de altitude é mostrada na Tabela 2 . As áreas de baixa altitude (cota abaixo de 300m) abrigam cerca de $65 \%$ da população total do estado e são responsáveis por $85,6 \%$ dos casos. As taxas de incidência da doença em maiores altitudes são significativamente inferiores à média do Estado.
A Tabela 3 mostra a incidência de leptospirose segundo bacias hidrográficas. Duas bacias, da Lagoa Mirim e do Rio Jacuí, apresentaram um número de casos significativamente acima do esperado. A maior parte das demais bacias apresentam valores significativamente inferior ao esperado, com exceção das bacias dos rios Pelotas e Taquari e do médio Uruguai, que apresentam taxas semelhantes à média de incidência no Estado.

Dos recortes espaciais utilizados, a classificação de unidades que apresentaram maior variabilidade não aleatória de resultados, isto é, 
Casos de leptospirose, população, taxa de incidência (casos por 100 mil habitantes) e área (em $\mathrm{km}^{2}$ ) em unidades de uso do solo no Estado do Rio Grande do Sul, Brasil.

\begin{tabular}{lcccc}
\hline Uso do solo & Número de casos & População & Taxa de incidência Área (km²) \\
\hline Vegetação secundária e atividades agrícolas & 509 & 3.712 .497 & 13,7 & 78.462 \\
Atividades agrícolas & 363 & 1.670 .808 & 21,71 & 48.580 \\
Savana & 336 & 3.406 .237 & 9,92 & 10.694 \\
Gramíneo-lenhosa & 55 & 926.827 & 5,92 & 93.078 \\
Influência marinha & 6 & 134.464 & 4,52 & 2.375 \\
Savana-Estepe & 3 & 196.390 & 1,52 & 10.932 \\
Parque & 2 & 79.538 & 2,52 & 12.767 \\
Arbórea aberta & 0 & 34.008 & 0,02 & 5.842 \\
Montanha & 0 & 27.029 & 0,0 & 2.239 \\
\hline
\end{tabular}

1 Taxa de incidência significativamente superior à média estadual segundo teste de Poisson.

2 Taxa de incidência significativamente inferior à média estadual segundo teste de Poisson.

Casos de leptospirose, população, taxa de incidência (casos por 100 mil habitantes)

e área (em km²) segundo faixas de relevo no Estado do Rio Grande do Sul, Brasil.

\begin{tabular}{lrrrrr}
\hline Faixa de altitude $(\mathbf{m})$ & Número de casos & População & Taxa de incidência Área (km²) \\
\hline 0 a 300 & 1.177 & 7.210 .189 & 16,31 & 164.645 \\
300 a 800 & 96 & 2.880 .340 & 3,32 & 90.811 \\
$>800$ & 1 & 97.269 & 1,02 & 13.626 \\
\hline
\end{tabular}

1 Taxa de incidência significativamente superior à média estadual segundo teste de Poisson.

2 Taxa de incidência significativamente inferior à média estadual segundo teste de Poisson.

com valores significativamente diferentes entre casos observados e esperados, foram a altitude, as bacias hidrográficas e o uso do solo, com valores de qui-quadrado de 144, 97 e 26, respectivamente. A divisão do estado por municípios não apresentou diferença significativa entre o número de casos esperados e observados, segundo valor global de qui-quadrado da distribuição.

A faixa de altitude controla a possibilidade de inundação, ao mesmo tempo em que condiciona a ocupação humana, especialmente mediante indução de atividades agrícolas mais adequadas. A importância das bacias hidrográficas como unidade de análise de processos sócio-ambientais tem sido ressaltada por entidades ambientais não governamentais e órgãos de governo. Em razão da característica de receptáculo de águas, diversos fatores ecológicos e geológicos são limitados pelas bacias hidrográficas. Além disso, a própria ocupação terri- torial do Rio Grande do Sul seguiu a delimitação de bacias hidrográficas, sendo marcante o primeiro ciclo de colonização açoreana no litoral e vale do Rio Jacuí; e de italianos e alemães, no final do século XIX, seguindo os vales dos rios Pardo, Taquari, Caí e Sinos, na encosta sul do Planalto Meridional. A fronteira seca com o Uruguai, na bacia de mesmo nome, foi dominada por grandes fazendas concedidas para a ocupação militar do território (Souza, 2000). Cada estratégia de ocupação correspondeu a um modelo de produção agrícola e deixou marcas culturais e fisiográficas nessas bacias (Rambo, 1994). Dessa maneira, as bacias hidrográficas representam uma síntese de processos históricos, ecológicos e políticos, além de constituir uma instância de gestão de recursos (Pereira, 2000).

A Figura 2 mostra as áreas do estado consideradas como críticas segundo as taxas de incidência de leptospirose calculadas. A sobreposi- 
Casos de leptospirose, população, taxa de incidência (casos por 100 mil habitantes)

e área (em km²) por bacia hidrográfica no Estado do Rio Grande do Sul, Brasil.

\begin{tabular}{lcccc}
\hline Bacia hidrográfica & Número de casos & População & Taxa de incidência Área (km²) \\
\hline Lagoa dos Patos & 615 & 5.369 .494 & 11,52 & 47.335 \\
Rio Jacuí & 262 & 1.299 .862 & 20,21 & 46.340 \\
Lagoa Mirim & 161 & 167.791 & 96,01 & 23.059 \\
Rio Taquari & 137 & 952.120 & 14,4 & 26.364 \\
Médio Uruguai & 61 & 634.955 & 9,6 & 32.312 \\
Alto Uruguai & 24 & 527.629 & 4,52 & 14.317 \\
Rio Ibicuí & 8 & 472.862 & 1,72 & 44.924 \\
Rio Passo Fundo & 4 & 259.183 & 1,52 & 8.282 \\
Litoral Norte & 1 & 76.947 & 1,32 & 2.554 \\
Rio Apuaé & 1 & 142.253 & 0,72 & 6.498 \\
Rio Quaraí & 0 & 154.822 & 0,02 & 8.902 \\
Rio Pelotas & 0 & 11.113 & 0,0 & 4.529 \\
Rio Negro & 0 & 118.767 & 0,02 & 3.177 \\
\hline
\end{tabular}

1 Taxa de incidência significativamente superior à média estadual segundo teste de Poisson.

2 Taxa de incidência significativamente inferior à média estadual segundo teste de Poisson.

ção dessas áreas demonstra a convergência de fatores ambientais de risco em algumas regiões do Estado, particularmente as localizadas na depressão central e ao longo do litoral da Lagoa Mirim. Essas são áreas de cultivo de arroz irrigado, sujeitas a inundações esporádicas. O trabalho nessas condições pode ser avaliado como um risco de infecção e adoecimento. São áreas caracterizadas pela presença de "banhados" ou brejos, sujeitos à inundação periódica, localizados à margem de rios. Algumas espécies de roedores existentes nos ecossistemas de banhado são a capivara (Hydrochoerus hydrochaeris) e ratão-do-banhado (Myocastor coypus) (Widholzer, 1986). Alguns estudos (SES-RS, 1999; Vinetz, 2001) têm mostrado a infecção desses animais pela leptospira e sua participação em ciclos silvestres da doença. Não deve ser descartado o papel de outros mamíferos como bois e cavalos na transmissão da leptospirose do meio rural (Barwick et al., 1998).

As áreas apontadas como críticas para a transmissão da leptospirose coincidem em grande parte com os municípios considerados de altas taxas de incidência. Contudo, a metodologia proposta por esse trabalho traz algumas diferenciações importantes que podem alterar as estratégias de controle da doença. Como o número de casos e a população de cada município são agregados em unidades espaciais maiores, os valores obtidos para taxas de incidência são razoavelmente mais robustos que as taxas originais por município. Tal trans- formação é particularmente importante no caso de um evento de saúde relativamente raro e para diagnósticos baseados em apenas um ano de observação. Em segundo lugar, a distribuição espacial de indicadores por unidades administrativas apresenta fortes descontinuidades, e é notável a presença alternada de municípios com baixa incidência no interior de áreas críticas de transmissão da doença. Esse é o caso de alguns municípios situados ao longo do litoral e na Depressão Central. Nesse caso, deve-se investigar a possível subnotificação da doença ou mesmo a presença de condições específicas de proteção da população. Esse detalhamento dos próximos passos de pesquisa e ação de vigilância é uma contribuição da abordagem proposta neste trabalho.

Alguns aspectos relativos à prevenção podem ser destacados com base na delimitação de áreas críticas. Um dos fatores de diferenciação da epidemiologia da leptospirose no Rio Grande do Sul é a fraca sazonalidade dos casos e a grande ocorrência de casos nas áreas rurais. Em diversos centros urbanos brasileiros, a leptospirose apresenta baixa endemicidade e eventuais surtos posteriores a enchentes, quando o sorovar L. icterohemorrhagiae é dominante (Ko et al., 1999).

Nas grandes áreas agrícolas, o ciclo de transmissão tem possivelmente outros sorovares envolvidos. Além disso, nessas áreas predominam outros animais como reservatório da leptospira. O controle das condições de trabalho no cam- 
Sobreposição de áreas críticas de incidência de leptospirose segundo faixa de altitude, bacias hidrográficas e uso do solo.

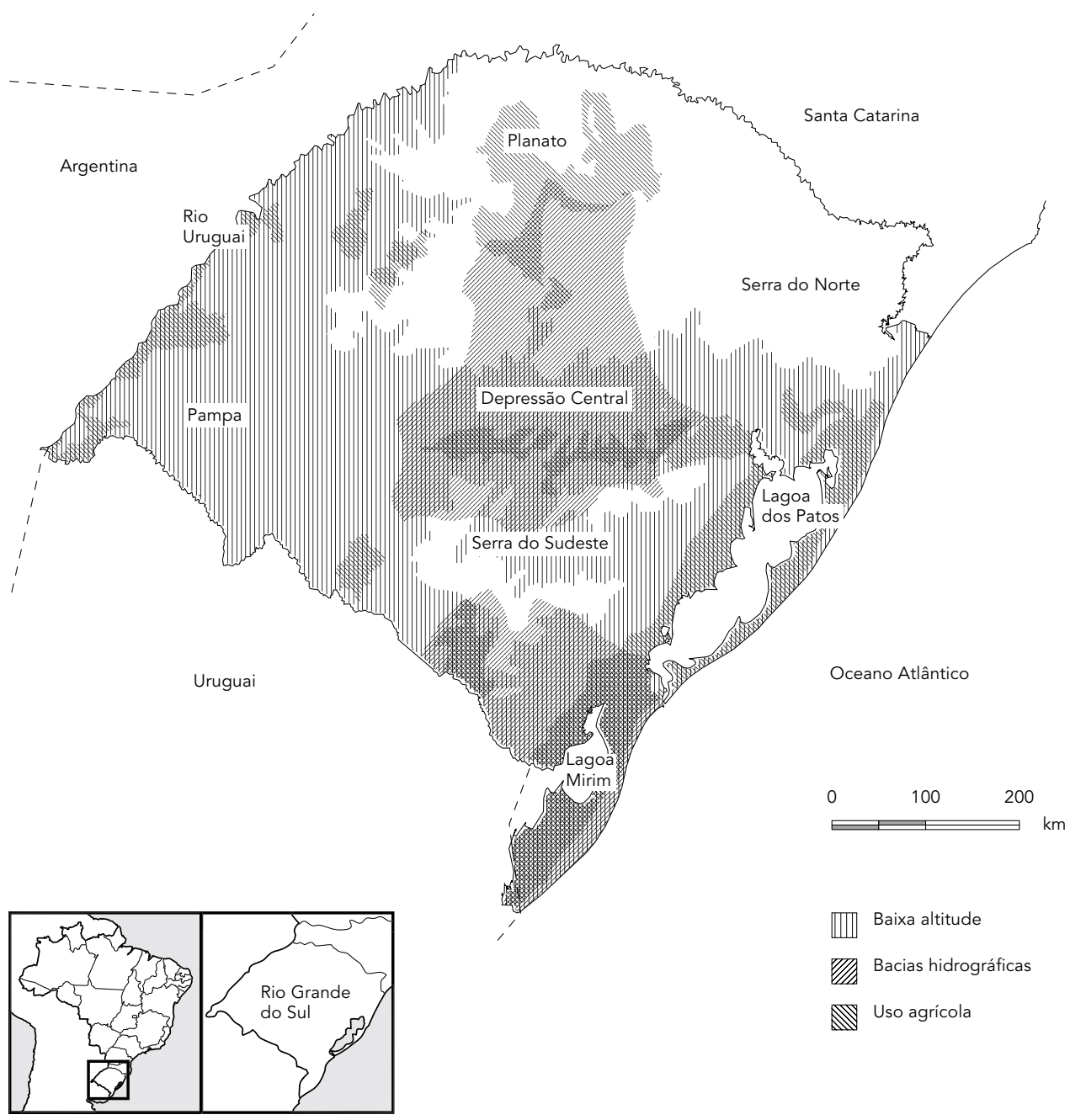

po reduziu a incidência de leptospirose em Israel. Atualmente o sorovar icterohemorrhagiae é dominante nas áreas urbanas e responsável pela maioria dos casos (Kariv et al., 2001). Os sorogrupos L. habdomadis e L. gryppotyphosa foram dominantes durante a década de 1970, sobretudo associados a atividades rurais. Nas ilhas Andaman e Nicobar, no Golfo de Bengala, os sorovares predominantes foram de L. australis e L. gryppotyphosa. A maior parte das ilhas é coberta por vegetação tropical úmida e plantações de arroz (Murhekar et al., 1998).

A fauna componente de um ecossistema está relacionada a processos ecológicos, biológi- cos e evolutivos (Hansson, 2002). Os roedores são animais prolíferos o que permite às populações efetuarem os ajustes necessários para enfrentar ambientes adversos. O controle das populações de roedores como o ratão-do-banhado em ambientes agrícolas é dificultado pela sua alta reprodutibilidade, que pode recolonizar áreas, retomando rapidamente as densidades originais. Logo, o controle da leptospirose no Rio Grande do Sul envolve a redução de fontes de alimento para esses roedores e a implantação de procedimentos de proteção aos trabalhadores dessas áreas. 


\section{Considerações finais}

A agregação de dados epidemiológicos em unidades espaciais de grande área e população permitiu a construção de taxas que representam importantes diferenciais entre áreas do estado. Ao mesmo tempo, essas taxas são mais robustas que os valores iniciais calculados para os municípios, que apresentaram uma grande aleatoriedade. A divisão por bacia hidrográfica apresentou uma grande variabilidade de taxas, demonstrando uma boa capacidade de diferenciar áreas de risco. As bacias são também importantes unidades de gestão ambiental, com a crescente implantação de comitês de bacias. Por outro lado, abrigam uma grande diversidade de paisagens. Outros recortes mostram a maior incidência em áreas agrícolas, de baixa altitude e úmidas. A combinação de camadas de informação que representam níveis de risco de transmissão da leptospirose é uma estratégia de recomposição de paisagens complexas (Kitron, 1998). A leptospirose, como outras doenças de forte influência de condições ambientais, possui múltiplos níveis de determinação. O estudo da sua distribuição espacial na escala regional permite a captação de fatores climáticos e ecológicos que atuam nesse nível, como as condições de sobrevivência de reservatórios.

\section{Referências}

ANEEL (Agência Nacional de Energia Elétrica), 2000. Sistema de Informações Georreferenciadas de Energia e Hidrologia. CD-ROM. Brasília: ANEEL.

BAILEY, T. C. \& GRATELL, A. C., 1995. Interactive Spatial Data Analysis. Essex: Longman Scientific \& Technical.

BARCELLOS, C. \& SANTOS, S. M., 1997. Colocando dados no mapa: A escolha da unidade espacial de agregação e integração de bases de dados em saúde e ambiente através do geoprocessamento. Informe Epidemiológico do SUS, 4:21-29.

BARWICK, R. S.; MOHAMMED, H. O. \& McDONOUGH, P. L., 1998. Epidemiologic features of equine Leptospira interrogans of human significance. Preventive Veterinarian Medicine, 36:153-165.

CARVALHO, M. S. \& CRUZ, O. G., 1998. Análise espacial por micro-áreas: Métodos e experiências. In: Epidemiologia: Contextos e Pluralidade (R. P. Veras, org.), pp. 79-89, Série Epidemiológica 4, Rio de Janeiro: Editora Fiocruz/ABRASCO.
A maior parte dos estudos ecológicos em epidemiologia utiliza áreas geográficas com unidade de análise, que representam recortes do espaço geográfico. Essas unidades são tomadas como observações independentes. No entanto, em diversas situações mediadas por fatores ambientais, as condições de saúde apresentam similaridades em áreas próximas entre si. Uma das soluções para se considerar a interação entre unidades espaciais tem sido a modelagem da estrutura espacial de dados valendo-se de unidades menores e a detecção de padrões a posteriori. Nesse caso, enquadra-se a maior parte das técnicas de estatística espacial de aglomerados (Nakaya, 2000). Neste trabalho, ao contrário, buscou-se identificar áreas de aglomerados com base na agregação em áreas construídas a priori, fundamentadas em critérios ambientais. O uso de municípios como unidade de agregação de dados apresentou grande variabilidade estatística do indicador. Além disso, tende a sobrevalorizar fatores locais em detrimento de macrocomponentes ecológicos. A abordagem ecossistêmica da saúde pública carece do desenvolvimento de metodologias capazes de identificar e agir sobre determinantes ambientais. A escolha de unidades espaciais de agregação de dados que melhor destaquem processos ambientais pode permitir a apreensão desses processos, que se verificam em escalas diferentes da divisão administrativa.

CLEEK, R. K., 1979. Cancer and the environment: The effect of scale. Social Science and Medicine, 13D: 241-247.

FORMAN, R. T. T. \& GODRON, M., 1986. Landscape Ecology. Nova York: John Wiley and Sons.

FUNASA (Fundação Nacional de Saúde), 2001. Manual de Controle da Leptospirose. Brasília: FUNASA.

HANSSON, L., 2002. Dynamics and trophic interactions of small rodents: Landscape or regional effects on spatial variation? Oecologia, 130:259-266.

IBGE (Fundação Instituto Brasileiro de Geografia e Estatística), 1986. Levantamento de Recursos Naturais. Volume 33 (Rio Grande do Sul). Rio de Janeiro: IBGE.

KARIV, R.; KLEMPFNER, R.; BARNEA, A.; SIDI, Y. \& SCHWARTZ, E., 2001. The changing epidemiology of leptospirosis in Israel. Emerging Infectious Diseases, 17:990-992.

KATZ, A. R.; MANEA, S. J. \& SASAKI, D. M., 1991. Lep- 
tospirosis on Kauai: Investigation of a common source of waterborne outbreak. American Journal of Public Health, 81:1310-1312.

KITRON, U., 1998. Landscape ecology and epidemiology of vector-borne diseases: Tools for spatial analysis. Journal of Medical Entomology, 35:435445.

KO, A. I.; GALVÃO-REIS, M.; RIBEIRO-DOURADO, C. M.; JOHNSON, W. D. \& RILEY, L. W., 1999. Urban epidemic of severe leptospirosis in Brazil. Lancet, 354:820-825.

KULLDORFF, M. \& NAGARWALLA, N., 1995. Spatial disease clusters: Detection and inference. Statistics in Medicine, 14:799-810.

LAURENT, M. L. \& THOMAS, I., 1997. Modèle d'interaction spatiale et agrégation des lieux. L'exemple des données criminelles. L'Espace Géographique, 26:269-279.

MILLS, J. N. \& CHILDS, J. E., 1998. Ecologic studies of rodent reservoirs: Their relevance for human health. Emerging Infectious Diseases, 4:529-537.

MONMONIER, M., 1996. How to Lie With Maps. 2nd Ed. Chicago: University of Chicago Press.

MORGAN, J.; BORNSTEIN, S. L.; KARPATI, A. M.; BRUCE, M.; BOLIN, C. A.; AUSTIN, C. C.; WOODS, C. W.; LINGAPPA, J.; LANGKOP, C.; DAVIS, B.; GRAHAM, D. R.; PROCTOR, M.; ASHFORD, D. A.; BAJANI, M.; BRAGG, S. L.; SHUTT, K.; PERKINS, B. A. \& TAPPERO, J. W., 2002. Outbreak of leptospirosis among triathlon participants and community residents in Springfield, Illinois. Clinical Infectious Diseases, 34:1593-1599.

MORGENSTERN, H., 1982. Uses of ecologic analysis in epidemiological research. American Journal of Public Health, 72:1336-1344.

MORRIS, R. D. \& MUNASINGHE, R. L., 1993. Aggregation of existing geographic region to diminish spurious variability of disease rates. Statistics in Medicine, 12:1915-1929.

MURHEKAR, M. V.; SUGUNAN, A. P.; VIJAYACHARI, P.; SHARMA, S. \& SEHGAL, S. C., 1998. Risk factors in the transmission of leptospiral infection. Indian Journal of Medical Research, 107:218-223.

NAKAYA, T., 2000. An information statistical approach to the modifiable areal unit problem in incidence rate maps. Environment and Planning A, 32:91101.
NURMINEN, M., 1995. Linkage failures in ecological studies. World Health Statistic Quarterly, 48:78-84.

OPENSHAW, S., 1984. Ecological fallacies and the analysis of areal census-data. Environment and Planning A, 16:17-31.

PEREIRA, P. A. S., 2000. Rios, Redes e Regiões: A Sustentabilidade a partir de um Enfoque Integrado de Recursos Terrestres. Porto Alegre: Editora Age.

PIQUET, R.; RANDOLPH, R.; SMOLKA, M. \& VETTER, D., 1986. Análise das Articulações Sócio-econômicas Regionais: Sugestões Metodológicas. Rio de Janeiro: Programa de Pós-Graduação em Planejamento Urbano e Regional, Universidade Federal do Rio de Janeiro.

RAMBO, B., 1994. A Fisionomia do Rio Grande do Sul. 3a Ed. São Leopoldo: Editora Unisinos.

SANTOS, M., 1999. A Natureza do Espaço: Técnica e Tempo, Razão e Emoção. São Paulo: Editora Hucitec.

SCHWARTZ, S., 1994. The fallacy of the ecological fallacy: The potential misuse of a concept and the consequences. American Journal of Public Health, 84:819-824.

SES-RS (Secretaria Estadual de Saúde do Rio Grande do Sul), 1999. Avaliação da Soroprevalência de Leptospirose no Ratão-do-Banhado. Relatório Técnico. Porto Alegre: Centro de Zoonoses e Vetores, SES-RS.

SOUZA, C. F., 2000. Contrastes Regionais e Formações Urbanas. Porto Alegre: Editora da UFRGS.

SUSSER, M., 1994a. The logic in ecological: I. The logic of analysis. American Journal of Public Health, 84: 825-829.

SUSSER, M., 1994b. The logic in ecological: II. The logic of design. American Journal of Public Health, 84:830-835.

VINE, M. F; DEGNAN, D. \& HANCHETTE, C., 1997. Geographic Information Systems: Their use in environmental epidemiologic research. Environmental Health Perspectives, 105:598-605.

VINETZ, J. M., 2001. Leptospirosis. Current Opinion in Infectious Disesases, 14:527-538.

WIDHOLZER, F. L., 1986. Banhados. Rio de Janeiro: Editora Imprinta.

Recebido em 25 de setembro de 2002

Versão final reapresentada em 23 de janeiro de 2003 Aprovado em 30 de abril de 2003 